\title{
Constraint Programming Based Production Planning in a Petrochemical Industry
}

\author{
Rajasekhar Kadambur and Prakash Kotecha
}

\begin{abstract}
In this article, we provide a constraint programming based optimization model to determine the optimal production planning to maximize profit in a petrochemical industry. The proposed model despite its simplicity overcomes the limitations of the mixed integer linear programming model available in literature. The benefits of the proposed model are demonstrated on various instances of two case studies and it is shown that the proposed formulation enables an increase in the profit in the range of 0.58 to $3.68 \%$.
\end{abstract}

Index Terms-Combinatorial optimization, constraint programming, petrochemical industry, production planning.

\section{INTRODUCTION}

A petrochemical industry uses series of complex networks to convert feedstock such as oil and gas to produce primary petrochemicals such as methanol, ethylene, propylene, benzene, toluene, xylene, etc. These primary petrochemicals are subsequently converted into petrochemical intermediates and derivatives which are ultimately transformed into products used in the market. The petrochemical industry operates at very high production levels which is achieved either through the large scale of the individual equipment or through the large scale of the entire plant [1]. A variety of optimization models have been proposed for efficiently operating the petrochemical plants. These include efficient production planning [2], mergers and acquisitions [1], integration of refineries and petrochemical plants [3], capacity expansion [4], efficient spatial organization of petrochemical plants [5], efficient job scheduling [6], and optimal supply chain management [7].

In this article, we use Constraint Programming (CP), an optimization technique that has its origins in the Artificial Intelligence and Computer Science Community [8], for solving the production planning problem in an industry. A Mixed Integer Linear Programming (MILP) model has been proposed in literature [2] to determine efficient production plans to guide the petrochemical industry development in Saudi Arabia. However, this formulation restricts the productions to 'artificial' levels thereby leading to suboptimal profit. In this article, we propose a $\mathrm{CP}$ based optimization formulation which overcomes the drawbacks of the MILP formulation in literature. The benefit of the proposed formulation has been demonstrated on eight different instances involving two distinct requirements. In the next

Manuscript received November 6, 2013; revised January 18, 2014

The authors are with the Department of Chemical Engineering, Indian Institute of Technology Guwahati, - 781 039, Assam, India (e-mail: r.kadambur@iitg.ernet.in, pkotecha@iitg.ernet.in). section, we provide a brief description of $\mathrm{CP}$ and this is followed by the description of the problem statement. In the next section, we review the MILP formulation in literature and describe its limitations. In the subsequent section, we present a $\mathrm{CP}$ based optimization formulation to solve the production planning problem and subsequently demonstrate the applicability of this formulation on various instances of two scenarios. We conclude the article by discussing the developments in this article and present possible future work in this direction.

\section{CONSTRAINT PROGRAMMING}

$\mathrm{CP}$ was predominantly used to solve Constraint Satisfaction Problems (CSP) and has been extended to solve single [9], [10] and multi objective optimization problems [11]. CP has found widespread application in solving combinatorial optimization [12]-[14] such as Assignment Problems [15], Network Problems [16], [17], Production Planning/Scheduling, Personnel Allocation. The benefits of $\mathrm{CP}$ include the ease of modeling as it does not restrict the user like mathematical programming, guarantee of the optimality of the solution even for non linear discrete problems and the ease of determination of value added solutions such as realizations and K-best solutions. Moreover, unlike other AI based optimization algorithms such as Genetic Algorithms, Simulated Annealing, Teaching Learning Based Optimization, it does not require any set of parameters that need to be tuned.

$\mathrm{CP}$ is an implicit, tree-based enumeration technique that intelligently uses the propagation of constraints to reduce the domain of the variables. Unlike optimization problems, the objective in CSP is to determine a feasible solution which is a set of singleton values for all the decision variables from its individual domains, while satisfying all the given constraints. In the initial phase, $\mathrm{CP}$ uses the constraints to eliminate as many infeasible combinations as possible by propagating the constraints. If it happens that a feasible solution is discovered in this phase, the procedure is terminated. However, it is very rare that this phase would lead to a feasible solution. Under such circumstances, a non deterministic choice is made on any of the decision variables i.e., a decision variable is fixed to one of the possible values from its domain. This procedure is referred to as choice point in CP literature. Subsequently, the constraints are propagated to reduce the domain of the other variables based on this choice point. If all the variables become singleton, the choice point is said to have led to a 'success' as a feasible solution is achieved. However, if the choice point leads to a situation where in the domain of at least one of the variables becomes empty, it is said to have led to a 'failure' and hence a revision of the choice point is 
necessary. It can be noted that at times, the selection of a single choice point is not sufficient and in such cases, a series of choice points are made till a failure or success is achieved. In case of failure, the latest choice point can be revised. Thus the selection of choice point and constraint propagation is carried repeatedly till a feasible solution is obtained.

$\mathrm{CP}$ solves optimization problems by transforming it into a constraint satisfaction problem by converting the objective function into a constraint with an appropriate bound. With the discovery of every feasible solution, the bound of the objective constraint is revised so as to determine an improved solution. This process is continued till the search space is completely explored or the problem becomes infeasible. The last updated bound corresponds to the globally optimal solution of the optimization problem. A detailed explanation on various aspects can be obtained from literature [8].

The drawbacks of CP include lack of use of relaxations and other geometrical properties of the problem to guide its search to the optimal solution. This at times makes $\mathrm{CP}$ computationally expensive for certain problems as it very often requires considerable computational resources to confirm the optimality of the solution. Many of the software restrict the use of $\mathrm{CP}$ to discrete variables and enforce various limitations on the use of continuous variables. Recent trends to overcome these drawbacks include the development of hybrid algorithms [18-20] which harness the complimentary benefits of $\mathrm{CP}$ and mathematical programming techniques.

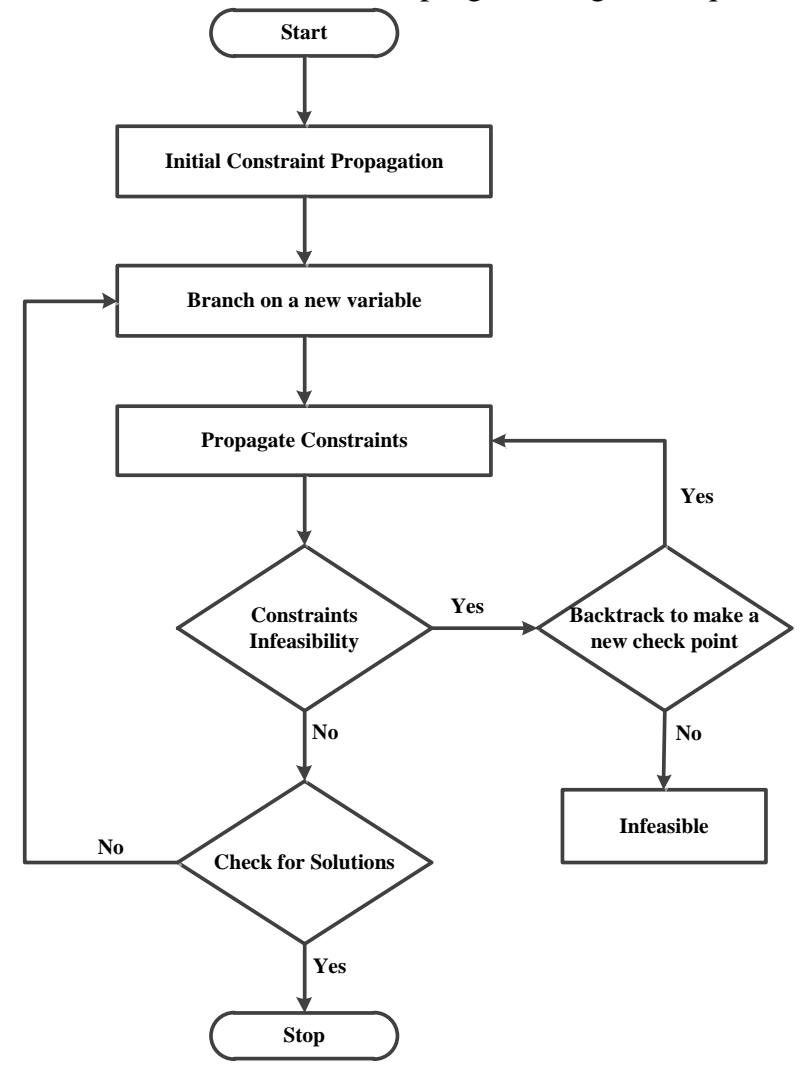

Fig. 1. Schematic of CP algorithm.

\section{PROBLEM DESCRIPTION}

The production planning problem is combinatorial in nature and involves deciding the quantities of $I$ products that need to be produced from a given set of $J$ processes so as to maximize the profit. Each of the process is assumed to produce only one product while some of the processes can produce the same product by using a specified amount of raw material. Each of these processes can be operated at low, medium or high level based on the amount of production. The production and investment cost of each of the processes depends on the amount of product (thus the production levels) that is produced form a particular process. The petrochemical industry has limited investment budget and has limited supply of raw materials thereby restricting the amount of products that can be produced. The production cost and investment cost are usually modeled as piecewise linear functions between the production levels as shown in Fig. 1.

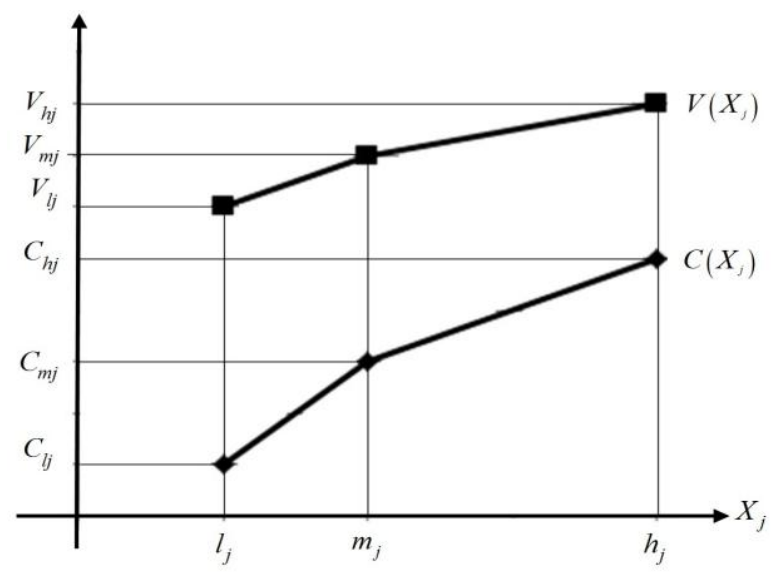

Fig. 2. Production and Investment cost varies nonlinearly with respect to capacity of production levels.

In addition to the constraints discussed above, sometimes a constraint is enforced that no product should be produced by more than one process. This requirement is known as the 'unique process requirement'. Table I and Table II shows an example of one such problem wherein 54 processes are available which can be operated at three different levels and can produce a maximum of 24 products. Without loss of generality, we have used this data for demonstrating the results of this work. In Table I, $N_{T}$ denotes the name of the product whereas $N_{S}$ indicates the name of the process. The notations $l_{j}, m_{j}$ and $h_{j}$ represent the units of production of the corresponding products in the low level, medium level and high level production capacity. The amount of raw material $\mathrm{R}_{1}$ and $\mathrm{R}_{2}$ required by the processes for producing per unit of the product is given by $b_{j 1}$ and $b_{j 2}$. The per unit sale price of the product is given by $E_{j}$. As can be seen from Table I, Product $F$ can be produced from six different processes (Process 10 to Process 15) and each of these processes have a different raw material requirement, production cost and investment cost that vary with the three different levels of production. If the unique processes requirement is not included, this product may be produced by more than one process of the six possible processes whereas if the unique process requirement is included, the Product $F$ is to be produced by only one of the six processes. It should be obvious that the inclusion of this constraint can lead to the reduction in profit. Some of the products in the Table I such as Process $19-24$, have zero requirement of raw material $R_{1}$ and $R_{2}$. It should be noted that these products use some other raw materials which do not have an upper limit in the availability. 
If upper limits on these raw materials exist, they too would have to be accommodated in the model formulation.

TABLE I: PRODUCTION LEVELS OF PROCESSES

\begin{tabular}{|c|c|c|c|c|c|c|c|}
\hline$\overline{N_{T}}$ & $\bar{N} N_{S}$ & $b_{j 1}$ & $b_{j 2}$ & $\overline{E E_{j}}$ & $\bar{l} l_{j}$ & $m_{j}$ & $\overline{h_{j}}$ \\
\hline \multirow{3}{*}{ A } & 1 & 0 & 3 & 3 & 35 & 44 & 91 \\
\hline & 2 & 0 & 2 & 5 & 38 & 55 & 93 \\
\hline & 3 & 0 & 2 & 6 & 13 & 69 & 84 \\
\hline \multirow{2}{*}{ B } & 4 & 0 & 3 & 5 & 38 & 51 & 73 \\
\hline & 5 & 0 & 2 & 7 & 29 & 58 & 77 \\
\hline \multirow{2}{*}{ C } & 6 & 0 & 5 & 6 & 13 & 47 & 98 \\
\hline & 7 & 0 & 1 & 5 & 18 & 63 & 75 \\
\hline $\mathrm{D}$ & 8 & 0 & 1 & 3 & 26 & 48 & 95 \\
\hline $\mathrm{E}$ & 9 & 0 & 1 & 6 & 39 & 56 & 87 \\
\hline \multirow{6}{*}{$\mathrm{F}$} & 10 & 0 & 4 & 2 & 39 & 61 & 10 \\
\hline & 11 & 0 & 3 & 4 & 14 & 67 & 73 \\
\hline & 12 & 0 & 4 & 2 & 40 & 69 & 84 \\
\hline & 13 & 0 & 1 & 4 & 39 & 57 & 74 \\
\hline & 14 & 0 & 5 & 7 & 25 & 45 & 99 \\
\hline & 15 & 0 & 4 & 2 & 34 & 45 & 71 \\
\hline \multirow{2}{*}{$\mathrm{G}$} & 16 & 0 & 2 & 7 & 14 & 48 & 94 \\
\hline & 17 & 0 & 4 & 5 & 23 & 66 & 95 \\
\hline $\mathrm{H}$ & 18 & 0 & 1 & 7 & 38 & 48 & 97 \\
\hline \multirow{2}{*}{ I } & 19 & 0 & 0 & 5 & 34 & 65 & 73 \\
\hline & 20 & 0 & 0 & 4 & 39 & 48 & 82 \\
\hline \multirow{2}{*}{$\mathrm{J}$} & 21 & 0 & 0 & 2 & 30 & 68 & 78 \\
\hline & 22 & 0 & 0 & 6 & 11 & 51 & 95 \\
\hline \multirow{3}{*}{$\mathrm{K}$} & 23 & 0 & 0 & 5 & 36 & 46 & 83 \\
\hline & 24 & 0 & 0 & 5 & 38 & 48 & 98 \\
\hline & 25 & 1 & 0 & 2 & 31 & 59 & 76 \\
\hline $\mathrm{L}$ & 26 & 1 & 0 & 4 & 33 & 55 & 78 \\
\hline $\mathrm{M}$ & 27 & 1 & 0 & 3 & 33 & 51 & 75 \\
\hline \multirow{3}{*}{$\mathrm{N}$} & 28 & 1 & 0 & 4 & 22 & 65 & 75 \\
\hline & 29 & 4 & 0 & 2 & 30 & 58 & 97 \\
\hline & 30 & 1 & 0 & 5 & 15 & 57 & 88 \\
\hline \multirow{3}{*}{$\mathrm{O}$} & 31 & 5 & 0 & 3 & 31 & 68 & 87 \\
\hline & 32 & 4 & 0 & 5 & 10 & 49 & 75 \\
\hline & 33 & 4 & 0 & 6 & 18 & 63 & 96 \\
\hline $\mathrm{P}$ & 34 & 3 & 0 & 4 & 11 & 63 & 89 \\
\hline \multirow{3}{*}{$\mathrm{Q}$} & 35 & 0 & 0 & 3 & 13 & 52 & 81 \\
\hline & 36 & 0 & 0 & 7 & 35 & 58 & 86 \\
\hline & 37 & 0 & 0 & 4 & 31 & 43 & 83 \\
\hline \multirow{3}{*}{$\mathrm{R}$} & 38 & 0 & 0 & 6 & 19 & 42 & 73 \\
\hline & 39 & 0 & 0 & 2 & 39 & 56 & 78 \\
\hline & 40 & 0 & 0 & 2 & 11 & 64 & 74 \\
\hline \multirow{2}{*}{$S$} & 41 & 0 & 0 & 5 & 23 & 69 & 76 \\
\hline & 42 & 0 & 0 & 7 & 21 & 44 & 78 \\
\hline \multirow{3}{*}{$\mathrm{T}$} & 43 & 0 & 0 & 2 & 33 & 58 & 83 \\
\hline & 44 & 0 & 0 & 4 & 34 & 55 & 72 \\
\hline & 45 & 0 & 0 & 6 & 15 & 41 & 98 \\
\hline \multirow{4}{*}{$\mathrm{U}$} & 46 & 4 & 0 & 7 & 25 & 51 & 99 \\
\hline & 47 & 3 & 0 & 3 & 23 & 45 & 85 \\
\hline & 48 & 3 & 0 & 7 & 30 & 64 & 85 \\
\hline & 49 & 2 & 0 & 4 & 31 & 50 & 81 \\
\hline \multirow{2}{*}{ V } & 50 & 0 & 0 & 7 & 33 & 56 & 98 \\
\hline & 51 & 0 & 0 & 2 & 18 & 45 & 82 \\
\hline $\mathrm{W}$ & 52 & 0 & 0 & 2 & 31 & 59 & 74 \\
\hline \multirow{2}{*}{$X$} & 53 & 0 & 0 & 4 & 30 & 48 & 94 \\
\hline & 54 & 0 & 0 & 2 & 15 & 60 & 82 \\
\hline
\end{tabular}

This problem has been previously modeled as a MILP but this formulation enforces that either the production from a process is between $l_{j}$ and $m_{j}$ or between $m_{j}$ and $h_{j}$ thereby preventing production using multiple levels. This MILP formulation also restricts the production of a product to $h_{j}$ which leads to suboptimal solutions as an 'artificial' limit is implemented on a highly profitable product.

TABLE II: PRODUCTION AND INVESTMENT COSTS

\begin{tabular}{|c|c|c|c|c|c|c|c|}
\hline$N_{T}$ & $N_{S}$ & $C l_{j}$ & $\mathrm{Cm}_{j}$ & $C h_{j}$ & $V l_{j}$ & $V m_{j}$ & $V h_{j}$ \\
\hline \multirow{3}{*}{ A } & 1 & 85 & 103 & 244 & 135 & 162 & 350 \\
\hline & 2 & 91 & 125 & 239 & 144 & 195 & 347 \\
\hline & 3 & 41 & 153 & 198 & 69 & 237 & 297 \\
\hline \multirow{2}{*}{ B } & 4 & 91 & 117 & 183 & 144 & 183 & 271 \\
\hline & 5 & 73 & 131 & 188 & 117 & 204 & 280 \\
\hline \multirow{2}{*}{$\mathrm{C}$} & 6 & 41 & 109 & 262 & 69 & 171 & 375 \\
\hline & 7 & 51 & 141 & 177 & 84 & 219 & 267 \\
\hline $\mathrm{D}$ & 8 & 67 & 111 & 252 & 108 & 174 & 362 \\
\hline$E$ & 9 & 93 & 127 & 220 & 147 & 198 & 322 \\
\hline \multirow{6}{*}{$\mathrm{F}$} & 10 & 93 & 137 & 254 & 147 & 213 & 369 \\
\hline & 11 & 43 & 149 & 167 & 72 & 231 & 255 \\
\hline & 12 & 95 & 153 & 198 & 150 & 237 & 297 \\
\hline & 13 & 93 & 129 & 180 & 147 & 201 & 269 \\
\hline & 14 & 65 & 105 & 267 & 105 & 165 & 381 \\
\hline & 15 & 83 & 105 & 183 & 132 & 165 & 269 \\
\hline \multirow{2}{*}{$\mathrm{G}$} & 16 & 43 & 111 & 249 & 72 & 174 & 358 \\
\hline & 17 & 61 & 147 & 234 & 99 & 228 & 344 \\
\hline $\mathrm{H}$ & 18 & 91 & 111 & 258 & 144 & 174 & 370 \\
\hline \multirow{2}{*}{ I } & 19 & 83 & 145 & 169 & 132 & 225 & 257 \\
\hline & 20 & 93 & 111 & 213 & 147 & 174 & 310 \\
\hline \multirow[b]{2}{*}{$\mathrm{J}$} & 21 & 75 & 151 & 181 & 120 & 234 & 274 \\
\hline & 22 & 37 & 117 & 249 & 63 & 183 & 359 \\
\hline \multirow{3}{*}{$\mathrm{K}$} & 23 & 87 & 107 & 218 & 138 & 168 & 316 \\
\hline & 24 & 91 & 111 & 261 & 144 & 174 & 374 \\
\hline & 25 & 77 & 133 & 184 & 123 & 207 & 275 \\
\hline $\mathrm{L}$ & 26 & 81 & 125 & 194 & 129 & 195 & 287 \\
\hline $\mathrm{M}$ & 27 & 81 & 117 & 189 & 129 & 183 & 279 \\
\hline \multirow{3}{*}{$\mathrm{N}$} & 28 & 59 & 145 & 175 & 96 & 225 & 265 \\
\hline & 29 & 75 & 131 & 248 & 120 & 204 & 360 \\
\hline & 30 & 45 & 129 & 222 & 75 & 201 & 325 \\
\hline \multirow{3}{*}{$\mathrm{O}$} & 31 & 77 & 151 & 208 & 123 & 234 & 310 \\
\hline & 32 & 35 & 113 & 191 & 60 & 177 & 281 \\
\hline & 33 & 51 & 141 & 240 & 84 & 219 & 351 \\
\hline $\mathrm{P}$ & 34 & 37 & 141 & 219 & 63 & 219 & 323 \\
\hline \multirow{3}{*}{$\mathrm{Q}$} & 35 & 41 & 119 & 206 & 69 & 186 & 302 \\
\hline & 36 & 85 & 131 & 215 & 135 & 204 & 316 \\
\hline & 37 & 77 & 101 & 221 & 123 & 159 & 319 \\
\hline \multirow{3}{*}{$\mathrm{R}$} & 38 & 53 & 99 & 192 & 87 & 156 & 280 \\
\hline & 39 & 93 & 127 & 193 & 147 & 198 & 286 \\
\hline & 40 & 37 & 143 & 173 & 63 & 222 & 262 \\
\hline \multirow[b]{2}{*}{$\mathrm{S}$} & 41 & 61 & 153 & 174 & 99 & 237 & 265 \\
\hline & 42 & 57 & 103 & 205 & 93 & 162 & 298 \\
\hline \multirow{3}{*}{$\mathrm{T}$} & 43 & 81 & 131 & 206 & 129 & 204 & 304 \\
\hline & 44 & 83 & 125 & 176 & 132 & 195 & 263 \\
\hline & 45 & 45 & 97 & 268 & 75 & 153 & 381 \\
\hline \multirow{4}{*}{$\mathrm{U}$} & 46 & 65 & 117 & 261 & 105 & 183 & 375 \\
\hline & 47 & 61 & 105 & 225 & 99 & 165 & 325 \\
\hline & 48 & 75 & 143 & 206 & 120 & 222 & 306 \\
\hline & 49 & 77 & 115 & 208 & 123 & 180 & 304 \\
\hline \multirow{2}{*}{$\mathrm{V}$} & 50 & 81 & 127 & 253 & 129 & 198 & 366 \\
\hline & 51 & 51 & 105 & 216 & 84 & 165 & 313 \\
\hline $\mathrm{W}$ & 52 & 77 & 133 & 178 & 123 & 207 & 267 \\
\hline \multirow{2}{*}{$X$} & 53 & 75 & 111 & 249 & 120 & 174 & 358 \\
\hline & 54 & 45 & 135 & 201 & 75 & 210 & 298 \\
\hline
\end{tabular}

\section{CONSTRAINT PROGRAMMING FORMULATION}

In this section, we propose a $\mathrm{CP}$ formulation for the production planning problem described in the previous section. As can be seen in Fig. 2, there is a straight line 
governing the production cost between $l_{j}$ and $m_{j}$ and another straight line between $m_{j}$ and $h_{j}$. Each of these two lines can be represented in the slope-intercept form. In the formulation below, $P C m h M_{j}$ and $P C m h \mathrm{C}_{j}$ denote the values of slope and intercept respectively for the line connecting $m_{j}$ and $h_{j}$ whereas $P C \operatorname{lm} M_{j}$ and $P C \operatorname{lm} \mathrm{C}_{j}$ denote the values of slope and intercept respectively for the line connecting $l_{j}$ and $m_{j}$. Similar to the lines for production cost, there are two other lines governing the investment cost between the intervals of $\left(l_{j}, m_{j}\right)$ and $\left(m_{j}, h_{j}\right)$ for the $j^{\text {th }}$ process. In the formulation below, ICImM $M_{j}$ and $\operatorname{IC} \operatorname{lm} \mathrm{C}_{j}$ will denote the value of slope and intercept respectively for the investment cost line connecting $l_{j}$ and $m_{j}$ whereas $I C m h M_{j}$ and $I C m h C_{j}$ will denote the value of the slope and intercept respectively for the investment cost line connecting $m_{j}$ and $h_{j}$. The parameter $P_{i j}$ is a binary parameter with a value of 1 if the $i^{\text {th }}$ product is produced from the $j^{\text {th }}$ process.

$$
\begin{aligned}
& \text { Maximize: } \quad \text { Profit }=\sum_{j=1}^{J} E_{j} X_{j}-P C_{j} \\
& \text { s.t } \quad z \operatorname{lm}_{j}=\min \left(1, x \operatorname{lm}{ }_{j}\right) \quad \forall j=1,2, \ldots, J \\
& z m h_{j}=\min \left(1, x m h_{j}\right) \quad \forall j=1,2, \ldots, J \\
& X \operatorname{lm}_{j}=0 \|\left(X \operatorname{lm}_{j} \geq l_{j} \& X \operatorname{lm}_{j} \leq m_{j}\right) \quad \forall j=1,2, \ldots, J \\
& X m h_{j}=0 \|\left(X m h_{j} \geq m_{j} \& X m h_{j} \leq h_{j}\right) \forall j=1,2, \ldots, J \\
& P C m h_{j}=\left(P C m h M_{j}\right)\left(X m h_{j}\right)+\left(z m h_{j}\right)\left(P C m h \mathrm{C}_{j}\right) \\
& I C m h_{j}=\left(I C m h M_{j}\right)\left(X m h_{j}\right)+\left(z m h_{j}\right)\left(I C m h \mathrm{C}_{j}\right) \\
& P C \operatorname{lm}{ }_{j}=\left(P C \operatorname{lm} M_{j}\right)\left(X \operatorname{lm}_{j}\right)+\left(z \operatorname{lm}{ }_{j}\right)\left(P C \operatorname{lm} \mathrm{C}_{j}\right) \\
& I C \operatorname{lm}{ }_{j}=\left(\operatorname{IClm}_{j}\right)\left(X \operatorname{lm}_{j}\right)+\left(\operatorname{zlm}_{j}\right)\left(\operatorname{IClm} \mathrm{C}_{j}\right) \\
& X_{j}=X m h_{j}+X m h_{j} \\
& P C_{j}=P_{C l m}+P C m h_{j} \\
& I C_{j}=I C l m_{j}+I C m h_{j} \\
& \sum_{j=1}^{J} b_{j t} X_{j} \leq R_{t}, \forall t=1,2, \ldots, T \\
& \sum_{j=1}^{J} I C_{j} \leq B \\
& \sum_{j=1}^{J} P_{i j} X_{j} \leq D_{i} \quad \forall i=1,2, . ., I
\end{aligned}
$$

In the above formulation, $z \operatorname{lm}_{j}$ is a binary variable that will be 1 if $x m_{j}>0$ and 0 otherwise. Similarly $z m h_{j}$ is a binary variable that will be 1 if $X m h_{j}>0$ and 0 otherwise. $X l m_{j}$ denotes the amount of product produced from a production level between $l_{j}$ and $m_{j}$ whereas $x m h_{j}$ denotes the amount of product produced from a production level between $m_{j}$ and $h_{j}$. It can be thus seen that a product can be simultaneously produced from all the possible levels. Equation (4) ensures that $X l m_{j}$ is either 0 or between $l_{j}$ and $m_{j}$ where the production and investment cost values can be determined while Equation (5) ensures $X m h_{j}>0$ that is either 0 or is between $m_{j}$ and $h_{j}$. As shown in Equation (10), $X_{j}$ denotes the total amount of product that is produced from the $j^{\text {th }}$ process. $\mathrm{PClm}_{j}$ and $\mathrm{IClm}_{j}$ indicate the production cost and investment cost required for producing $X_{1} m_{j}$ and are given by Equation (8) and Equation (9) respectively. Similarly, $\mathrm{PCmh}_{j}$ and $\mathrm{ICmh}_{j}$ indicate the production and investment cost required to produce $X m h_{j}$ and are given by Equation (6) and Equation (7) respectively. The total production and investment cost of the $j^{\text {th }}$ process are given by $P C_{j}$ and $I C_{j}$ respectively and are determined by Equation (11) and Equation (12) respectively. Equation (14) ensures that the total investment cost is below the investment budget whereas Equation (13) ensures that the raw materials required for the production of is not greater than the amount of the particular raw material that is available. Equation (15) is used to ensure that the total production of the $i^{\text {th }}$ product from all the processes is less than the demand of the $i^{\text {th }}$ product. This constraint is implemented as in literature and can be easily modified according to the specific needs of the user. For example, if there is a penalty incurred if a demand is not satisfied, it may be more appropriate to ensure that the production is more than the demand. The domain of $X l m_{j}$ is restricted to integers in $\left(0, m_{j}\right)$ whereas the domain of $X m h_{j}$ is restricted to integers in $\left(0, h_{j}\right)$. In case of unique process requirement, the following two constraints are added to the above CP model

$$
\begin{array}{ll}
Z_{j}=\min \left(1, X_{j}\right) & \forall j=1,2, \ldots, J \\
\sum_{j=1}^{J} P_{i j} Z_{j} \leq 1 & \forall i=1,2, . ., I
\end{array}
$$

Thus the proposed model involves $3 J$ integer decision variables corresponding to $X l m_{j}, X m h_{j}$ and $X_{j}$ and $3 J$ binary decision variables corresponding to $z l m_{j}, z m h_{j}$ and $Z_{j}$. It can be seen that the above formulation is easy to build and understand. It uses the Boolean 'or' constraints denoted by the symbol || and the Boolean 'and' constraints denoted by the symbol \&. Such constraints can easily be accommodated due to the highly expressive modeling power of $\mathrm{CP}$ and are available in many of the CP based solvers. It is easy to note that the above formulation accurately determines the production cost and investment cost between the interval $\left(l_{j}, m_{j}\right)$ and $\left(m_{j}, h_{j}\right)$ using the corresponding line that governs it. Moreover in this proposed formulation, if profitable, the $j^{\text {th }}$ process can produce a product quantity greater than $h_{j}$, due to the incorporation of $X I m_{j}$ and $X m h_{j}$. It should be noted that, if desired, the above special constraints could be possibly converted using discrete variables into the conventional inequalities of the form $\leq$, = and $\geq$ to obtain a MILP formulation. However such a strategy including its computational complexity is beyond the scope of this work. Though the above formulation is postulated for the 
production planning in a petrochemical industry, it can also be used in the production planning of any multi-level production processes. Also, it can also be realized that the above formulation can be easily extend to accommodate more than three levels of production capacity.

\section{Results And DisCussions}

In this section, we demonstrate the benefits of the proposed $\mathrm{CP}$ formulation on two scenarios with each involving four different instances. Instance 1 to Instance 4 comprises Scenario I and it enforces the unique process requirement whereas Instance 5 to 8 comprises Scenario II and does not enforce the unique process requirement. The four instances in each case study differ in the amount of investment budget that is available for the investment. Both the raw materials in all that the eight instances are assumed to be 50 units.

TABLE III: OPTIMAL PROFIT AND RESOURCE UTILIZATION

\begin{tabular}{|c|c|c|c|c|c|}
\hline & & Item & $\mathbf{C P}$ & MILP & $\begin{array}{l}\% \text { Profit } \\
\text { Increase }\end{array}$ \\
\hline \multirow{16}{*}{ 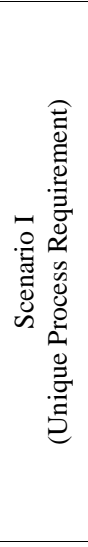 } & \multirow{4}{*}{1} & $B(\$)$ & 500 & 500 & \multirow{4}{*}{0.58} \\
\hline & & $R_{1}$ (units/yr) & 0 & 0 & \\
\hline & & $R_{2}$ ( units /yr) & 0 & 48 & \\
\hline & & Profit (\$/yr) & 642 & 638.3 & \\
\hline & \multirow{4}{*}{2} & $B(\$)$ & 650 & 650 & \multirow{4}{*}{2.38} \\
\hline & & $R_{1}$ ( units /yr) & 0 & 0 & \\
\hline & & $R_{2}$ ( units /yr) & 0 & 48.2 & \\
\hline & & Profit (\$/yr) & 859 & 839 & \\
\hline & \multirow{4}{*}{3} & $B(\$)$ & 750 & 750 & \multirow{4}{*}{0.81} \\
\hline & & $R_{1}$ ( units /yr) & 0 & 0 & \\
\hline & & $R_{2}$ ( units /yr) & 0 & 48 & \\
\hline & & Profit (\$/yr) & 990 & 982 & \\
\hline & \multirow{4}{*}{4} & $B(\$)$ & 798 & 800 & \multirow{4}{*}{3.68} \\
\hline & & $R_{1}$ (units/yr) & 0 & 0 & \\
\hline & & $R_{2}$ (units/yr) & 0 & 48 & \\
\hline & & Profit (\$/yr) & 1070 & 1032 & \\
\hline \multirow{16}{*}{ 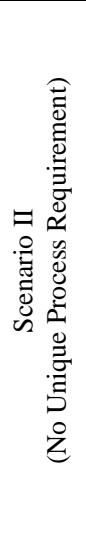 } & \multirow{4}{*}{5} & $B(\$)$ & 500 & 500 & \multirow{4}{*}{0.58} \\
\hline & & $R_{1}$ (units/yr) & 0 & 0 & \\
\hline & & $R_{2}$ (units/yr) & 0 & 48 & \\
\hline & & Profit (\$/yr) & 642 & 638.3 & \\
\hline & \multirow{4}{*}{6} & $B(\$)$ & 650 & 650 & \multirow{4}{*}{2.38} \\
\hline & & $R_{1}$ (units/yr) & 0 & 0 & \\
\hline & & $R_{2}$ (units/yr) & 0 & 48 & \\
\hline & & Profit (\$/yr) & 859 & 839 & \\
\hline & \multirow{4}{*}{7} & $B(\$)$ & 750 & 750 & \multirow{4}{*}{0.81} \\
\hline & & $R_{1}$ (units/yr) & 0 & 0 & \\
\hline & & $R_{2}$ (units/yr) & 0 & 48 & \\
\hline & & Profit (\$/yr) & 990 & 982 & \\
\hline & \multirow{4}{*}{8} & $B(\$)$ & 798 & 800 & \multirow{4}{*}{3.68} \\
\hline & & $R_{1}$ (units/yr) & 0 & 0 & \\
\hline & & $R_{2}$ (units/yr) & 0 & 48 & \\
\hline & & Profit (\$/yr) & 1070 & 1032 & \\
\hline
\end{tabular}

The results reported in this section were obtained using the default settings of IBM ILOG CPLEX Optimization Studio on a Processor Intel i5-2400 CPU @ 3.1 GHz. IBM ILOG CPLEX Optimization Studio allows the use of decision variables and decision expressions. To better utilize the computational resources, Equation (4)-(5), (13)-(17) are treated as constraints and are included in the "subject to" part of the model while Equation (2)-(3), (6)-(12) are considered as decision expressions.

Table III details the comparison of profit obtained using CP and the MILP formulation. It also provides the amount of raw materials and the amount of investment budget that is utilized in the corresponding production plan. In all the eight instances, it can be seen that $\mathrm{CP}$ is able to provide a better objective than the MILP formulation in literature. The increase in profit percentage ranges from 0.58 to 3.68. This increment in profit is due to the fact that the $\mathrm{CP}$ formulation permits higher production of a profitable product whereas the MILP formulation restricts the production of a profitable product to be less than or equal to $h_{j}$. This can be seen from the production plan provided in Table IV for all the eight instances. From Table IV, for Instance 1, it can be seen that the Process 36 is selected to produce 139 units of Product Q using both $x m_{j}$ and $x m h_{j}$ production levels. However from Table I, it can be seen that the value of $h_{j}$ for Process 36 is 86 and thus the MILP formulation cannot produce more than 86 units. This leads to suboptimal solution being provided by the MILP formulation in literature.

TABLE IV: PRODUCTION PLAN FOR OPTIMAL PROFIT

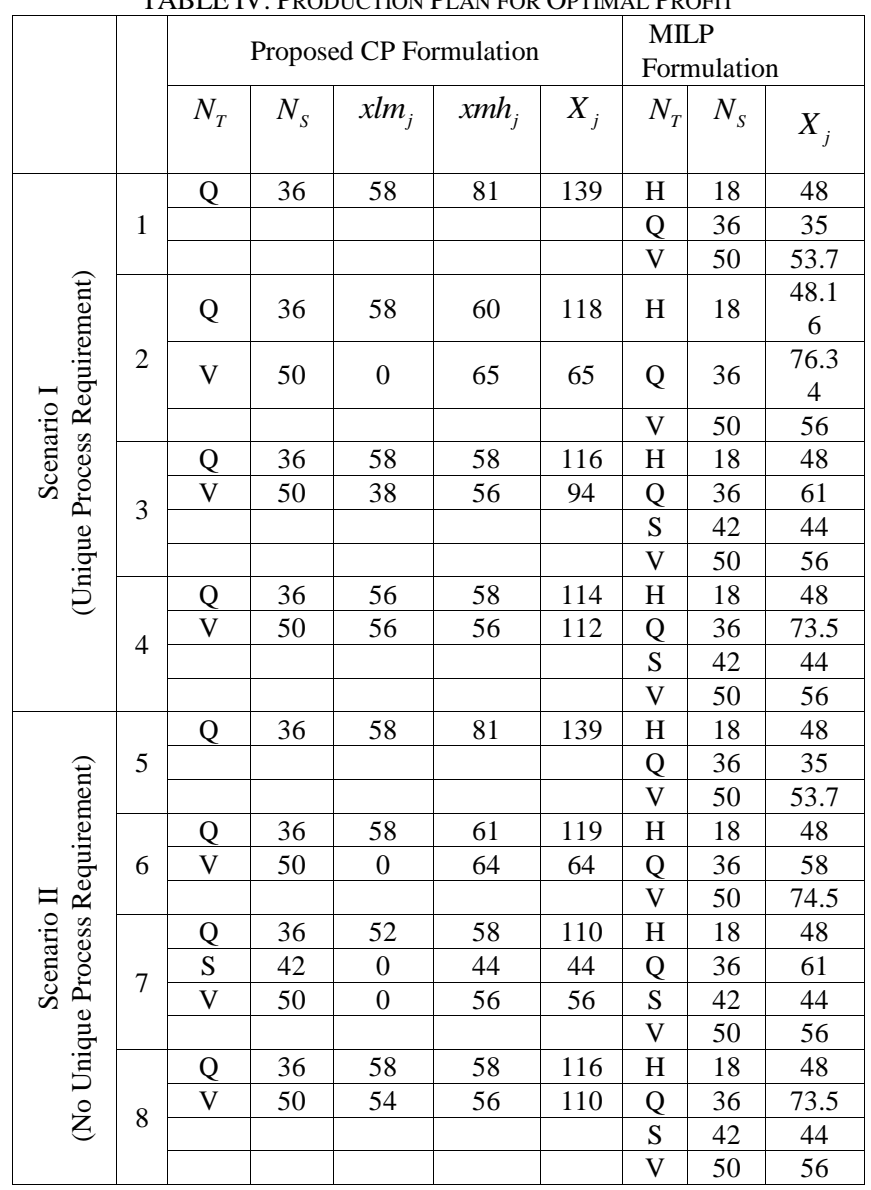

From Table IV for Instance 1, it can be seen that there are two units of Process 36 which are used to produce 139 units of Product Q since a single unit of $h_{j}$ cannot produce more than 86 . The complete budget of $\$ 500$ in this case is utilized and hence there is neither additional production of Product Q nor the production of any other product. For all the eight instances in Table IV, it can be observed that Product $\mathrm{Q}$ is produced from Process 36. A careful analysis of the data in Table I and Table II shows that the profit per unit of Product $Q$ is higher and it does not depend on either raw material $R_{1}$ or $R_{2}$ which are available in limited quantities. Thus the only restriction on the amount of Product Q that can be produced is due to the investment budget. The MILP formulation restricts the 
amount of Product Q that can be produced to $h_{j}$ whereas the CP formulation restricts the amount of Product $\mathrm{Q}$ that can be produced to $\left(m_{j}+h_{j}\right)$ which leads to higher profit.
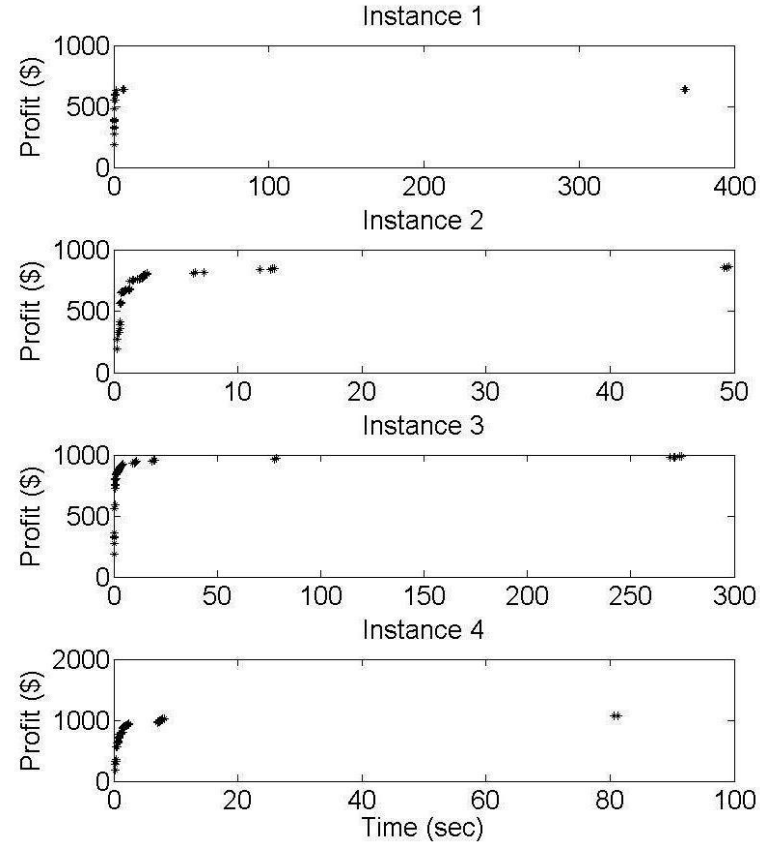

Fig. 3. Computational performance of CP for scenario I.
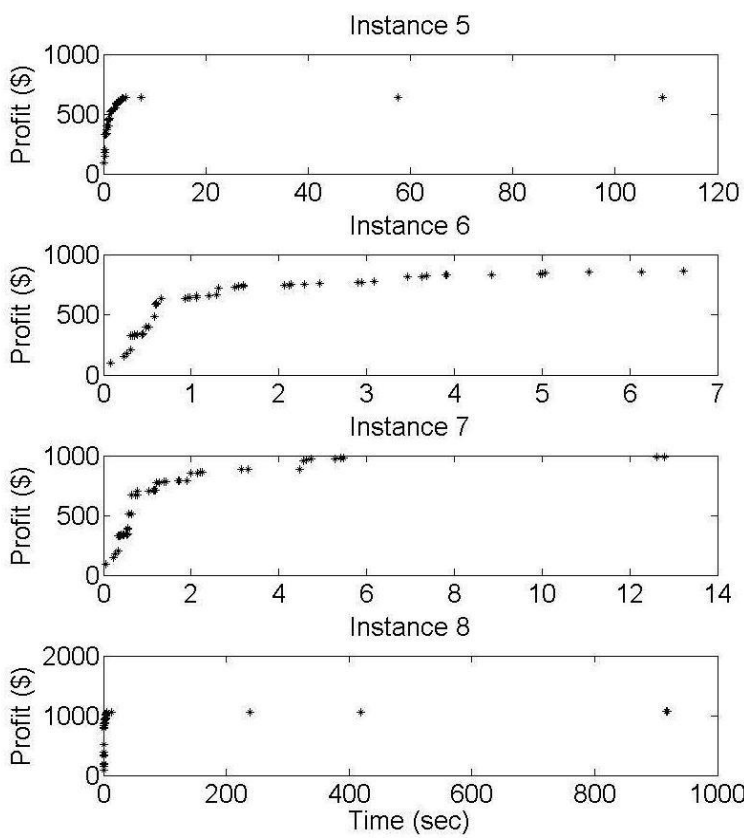

Fig. 4. Computational performance of CP for scenario II.

It can be seen that the profit in Instance 1 and Instance 5 provided by the $\mathrm{CP}$ formulation are identical and the production plans are also identical. However, for Instance 2 and Instance 6 , the profit provided by $\mathrm{CP}$ is identical but the production plans are not identical. Thus, these two production plans can be considered as realizations or multiple solutions as they have identical objective function value but have different sets of decision variables. Similarly, the CP solutions given in Instance 3 and Instance 7 are realizations similar to those between Instance 4 and Instance 8 . However, the MILP solutions in Instance 4 and Instance 8 are identical solutions similar to the pair of solutions in Instance 3 and
Instance 7. The computational performance of all the eight instances is shown in Fig. 3 and Fig. 4. It can be seen that CP is able to quickly determine the feasible solutions. The values reported in Table II correspond to the solutions determined by CP after 3600 seconds. It was observed that these solutions were obtained much earlier but there is no further improvement in the solutions provided by $\mathrm{CP}$. As mentioned earlier, this is a drawback of CP that it is computationally intensive, particularly to ensure global optimality. However, it should be noted that this is a design problem and it is not prohibitive to spend some computational effort to determine the profitable solutions. Moreover as the objective of this work is to provide an alternate formulation overcoming the drawbacks of the formulation in literature, additional techniques to direct the search and significantly improve the computational time are not explored in this article and will be a subject of future work.

\section{CONCLUSIONS}

In this article, we have proposed a constraint programming based model for determining the optimal production planning in a petrochemical industry. The proposed formulation overcomes the limitations of the MILP formulation in the literature and leads to better solutions. Future work can include incorporation of appropriate cuts and developing a hybrid strategy to reduce the computational efforts required by the proposed model. Additionally, other objectives that affect the petrochemical industries can be accommodated to determine efficient production plans in the midst of conflicting objectives.

\section{ACKNOWLEDGMENT}

The authors would like to acknowledge IBM Academic Initiative for providing the IBM ILOG CPLEX Optimization Studio software

\section{REFERENCES}

[1] S. G. Yoon, S. B. Park, S. Park, J. Lee, P. M. Verderame, and C. A Floudas, "Synergy in mergers of petrochemical companies within a complex considering purchasing and selling advantage with process integration," Industrial and Engineering Chemistry Research, vol. 47 no. 15 , pp. 5556-5567, 2008

[2] H. Alfares and A. A. Amer, "An optimization model for guiding the petrochemical industry development in Saudi Arabia," Engineering Optimization, vol. 34, no. 6, pp. 671-687, 2002.

[3] K. A. Qahtani and A. Elkamel, "Multisite refinery and petrochemical network design: optimal integration and coordination," Industrial \& Engineering Chemistry Research, vol. 48, no. 2, pp. 814-826, 2008.

[4] J. K. Bok, H. Lee, and S. Park, "Robust investment model for long-range capacity expansion of chemical processing networks under uncertain demand forecast scenarios," Computers \& Chemical Engineering, vol. 22, no. 7-8, pp. 1037-1049, 1998.

[5] H. Liu, F. Jin, Y. Liu, J. Ding, and X. Xu, "Evaluation and optimization of the spatial organization of the petrochemical industry in China," Journal of Geographical Sciences, vol. 23, no. 1, pp. 163-178, 2013.

[6] T. Lee, J. H. Ryu, I. B. Lee, and H. K. Lee, "A synchronized feed scheduling of petrochemical industries simultaneously considering vessel scheduling and storage tank management," Industrial \& Engineering Chemistry Research, vol. 48, no. 5, pp. 2721-2727, 2009.

[7] H. M. S. Lababidi, M. A. Ahmed, I. M. Alatiqi, and A. F. Al-Enzi, "Optimizing the supply chain of a petrochemical company under uncertain operating and economic conditions," Industrial \& Engineering Chemistry Research, vol. 43, no. 1, pp. 63-73, 2003 
[8] I. J. Lustig and J. F. Puget, "Program does not equal program: constraint programming and its relationship to mathematical programming," Interfaces, vol. 31, no. 6, pp. 29-53, 2001.

[9] I. E. Grossmann and L. T. Biegler, "Part II. Future perspective on optimization," Computers \& Chemical Engineering, vol. 28, no. 8, pp. 1193-1218, 2004.

[10] J. Hooker, Logic-Based Methods for Optimization: Combining Optimization and Constraint Satisfaction, Wiley, New York, 2000

[11] P. R. Kotecha, M. Bhushan, and R. D. Gudi, "Efficient optimization strategies with constraint programming," AIChE Journal, vol. 56, no. 2, pp. 387-404, 2010.

[12] S. Heipcke, "Comparing constraint programming and mathematical programming approaches to discrete optimisation-The change problem," The Journal of the Operational Research Society, vol. 50, no. 6 , pp. 581-595, 1999.

[13] L. Proll and B. Smith, "Integer linear programming and constraint programming approaches to a template design problem," Journal on Computing, vol. 10, no. 3, pp. 265-275, 1998.

[14] B. Smith, S. Brailsford, P. Hubbard, and H. P. Williams, "The progressive party problem: Integer linear programming and constraint programming compared," Principles and Practice of Constraint Programming, Springer Berlin Heidelberg, 1995.

[15] K. D. Dowman, J. Little, G. Mitra, and M. Zaffalon, "Constraint logic programming and integer programming approaches and their collaboration in solving an assignment scheduling problem," Constraints, vol. 1, no. 3, pp. 245-264, 1997.

[16] P. R. Kotecha, M. Bhushan and R. D. Gudi, "Constraint programming based robust sensor network design†," Industrial \& Engineering Chemistry Research, vol. 46, no. 18, pp. 5985-5999, 2007.
[17] P. R. Kotecha, M. Bhushan, and R. D. Gudi, "Design of robust, reliable sensor networks using constraint programming," Computers \& Chemical Engineering, vol. 32, no. 9, pp. 2030-2049, 2008.

[18] V. Jain and I. E. Grossmann, "Algorithms for hybrid MILP/CP models for a class of optimization problems," Journal on Computing, vol. 13 , no. 4, pp. 258-276, 2001.

[19] C. T. Maravelias and I. E. Grossmann, "A hybrid MILP/CP decomposition approach for the continuous time scheduling of multipurpose batch plants," Computers \& Chemical Engineering, vol. 28, no. 10, pp. 1921-1949, 2004

[20] B. Roe, L. G. Papageorgiou, and N. Shah, "A hybrid MILP/CLP algorithm for multipurpose batch process scheduling," Computers \& Chemical Engineering, vol. 29, no. 6, pp. 1277-1291, 2005.

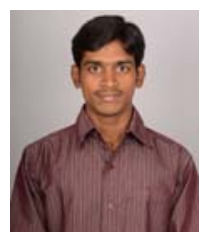

Rajasekhar Kadambur received his bachelor degree in chemical engineering from S.V. University in 2011. He is currently pursuing his $\mathrm{M}$. Tech in chemical engineering from Indian Institute of Technology Guwahati, Assam, India. His research interests include material science and applied optimization.

Prakash Kotecha received his bachelor degree in chemical engineering from Pondicherry Engineering College, Puducherry in 2002 and his doctoral degree from Indian Institute of Technology Bombay, Mumbai, India in 2010. $\mathrm{He}$ is currently working as a faculty in the Department of Chemical Engineering at Indian Institute of Technology Guwahati, Guwahati, Assam, India. 